\title{
Variation of diffusivity with the cation radii in molten salts of superionic conductors containing iodine anion: A molecular dynamics study ${ }^{\#}$
}

\author{
SRINIVASA R VARANASI and S YASHONATH* \\ Solid Sate and Structural Chemistry Unit, Indian Institute of Science, Bangalore 560 012, India \\ e-mail: yashonath@sscu.iisc.ernet.in
}

\begin{abstract}
A molecular dynamics study of the dependence of diffusivity of the cation on ionic radii in molten AgI is reported. We have employed modified Parinello-Rahman-Vashistha interionic pair potential proposed by Shimojo and Kobayashi. ${ }^{1}$ Our results suggest that the diffusivity of the cation exhibits an increase followed by a decrease as the ionic radius is increased. Several structural and dynamical properties are reported.
\end{abstract}

Keywords. diffusion; superionic conductor; molten salt.

\section{Introduction}

Superionic conductors are a class of materials which exhibit some properties characteristic of liquids while at the same time other properties are characteristic of solids. Several classes of materials are now known in which ionic motion is especially rapid. These materials are mainly classified into 'soft framework' and 'covalent framework'. The structural rigidity of the essentially immobile ion sub-lattice differ in these two materials. The interactions among the ions in soft frameworks are predominantly ionic and hence show lower melting points in comparison with covalent framework structures. The soft framework structures include two kinds of cubic structures, those in which the anionic arrangement is body-centered cubic (b.c.c.) (e.g., $\alpha-\mathrm{AgI})$, and the other in which anions are arranged in f.c.c. packing such as (e.g., $\alpha$-CuI). ${ }^{2}$ The principal aim of most of the studies on these systems is to understand the origin of the high ionic conductivity. For more than two decades, silver iodide(AgI) and copper iodide $(\mathrm{CuI})$ have been widely studied both theoretically and experimentally. ${ }^{3-11}$

The early work of Vashistha, Rahman and Parinello on $\mathrm{AgI}$ has led to number of studies in the field of computer simulation of superionic solids. ${ }^{4,12-14}$ Following their strategy, several researchers have investigated other soft frameworks like $\mathrm{Ag}_{2} \mathrm{~S}, \mathrm{Ag}_{2} \mathrm{Se}, \mathrm{Ag}_{2} \mathrm{Te}$, $\mathrm{CaF}_{2}$ etc. ${ }^{8,15,16}$ Another group of superionic solids are covalent framework solids. These frameworks consist of interconnected polyhedra, often tetrahedra or octahedra or both. The $\beta$-aluminas $\left(\mathrm{M}_{2} \mathrm{O} \cdot \mathrm{xAl}_{2} \mathrm{O}_{3}\right.$,

\footnotetext{
\#Dedicated to Prof. N Sathyamurthy on his 60th birthday

*For correspondence
}

$\mathrm{M}=\mathrm{Li}, \mathrm{Na}, \mathrm{Ag}$ etc. $)$, NASICON $\left(\mathrm{Na}_{1+x} \mathrm{Zr}_{2} \mathrm{Si}_{x} \mathrm{P}_{3-x} \mathrm{O}_{12}\right)$, pyrochlore $\left(\mathrm{MSbO}_{3}, \mathrm{M}=\mathrm{Na}\right.$ and $\left.\mathrm{K}\right)$ are examples of superionic solids with covalent frameworks. ${ }^{17}$

Flygare and Huggins in their study of superionic solids suggest that the fast ionic conduction arises from the existence of tunnels defined by the presence of the anions. They have carried out a computational study with anions $\left(\mathrm{I}^{-}\right)$located on a b.c.c. lattice and vary the radius of the cation. They computed minimum energy pathway and activation energy. They find that the activation energy is lowest for cations of intermediate size. ${ }^{18}$ Rahman and co-workers ${ }^{19,20}$ have proposed an inter-ionic pair potential function (PRV potential) to carry out molecular dynamics simulations on $\alpha$-AgI. They have successfully demonstrated the structural phase transition $(\beta \leftrightarrow \alpha)$ in $\mathrm{AgI}$, through their model potential function by using variable shape molecular dynamics technique. ${ }^{21}$ They have extensively studied cation behaviour in $\alpha$-AgI and succeeded in interpreting inelastic neutron scattering data. But the average density they obtained from their simulations is $5.474 \mathrm{gm} / \mathrm{cc}$ at $495 \mathrm{~K}$, whereas the experimental density is $5.99 \mathrm{gm} / \mathrm{cc}$. Later, Shimojo and Kobayashi. ${ }^{1}$ modified the parameters given by Parinello et al. ${ }^{20}$ to obtain the correct mass density. They performed a systematic study on both $\alpha$ and molten phases of AgI. They considered the same effective pair potentials to study both the phases.

Following the strategy of Rahman and co-workers, Padmanabhan and Yashonath have constructed an interionic potential function for NASICON type materials. Using this, they have studied several dynamical and structural properties of NASICON type materials using molecular dynamics simulations. The proposed interionic potential has been found to predict known 
quantities relating to structure, conductivity and other properties in good agreement with experiments. ${ }^{22}$ Their investigation suggests that there exists a particular radius of the mobile cation for which the diffusivity is maximum. This anamolous behaviour in the diffusion of the ion is known as 'Levitation Effect'. ${ }^{23}$ This effect was initially observed in their studies on diffusion of hydrocarbons in zeolite cages. Subsequently, this effect has been seen in many other condensed matter phases suggesting that it is an universal effect. ${ }^{24}$ It has been seen that the diffusant of the size corresponding to diffusivity maximum shows a lower activation energy for diffusion as compared to diffusants of sizes away from the diffusivity maximum. ${ }^{24}$

Roselieb and Jambon have conducted an experimental study on tracer diffusion of alkaline earth metal ions such as $\mathrm{Mg}^{2+}, \mathrm{Ca}^{2+}, \mathrm{Sr}^{2+}$ and $\mathrm{Ba}^{+2}$ in sodium aluminosilicate melts. Their findings reveal that both $\mathrm{Ca}^{2+}$ and $\mathrm{Sr}^{2+}$ show higher diffusivity as well as lower activation energy as compared to $\mathrm{Mg}^{2+}$ despite its smaller ionic radius. ${ }^{25}$ Huang et al. ${ }^{26}$ have studied the ionic diffusion in $\mathrm{SiO}_{2}$ melt as a function pressure and found that there is a marked increase in diffusivity of both $\mathrm{Si}$ and $\mathrm{O}$. They found a maximum in diffusivity at a pressure of $10 \mathrm{GPa}$. They related this behaviour with an increase in three-fold and five-fold coordination of Si-O with pressure.

Here we report an equilibrium microcanonical ensemble molecular dynamics (MD) study of dependence of diffusivity of $\mathrm{Ag}^{+}$on its ionic radius in molten $\mathrm{AgI}$ at $1000 \mathrm{~K}$. The aim of the present study is to investigate if the levitation effect exists and study its characteristics. The results suggest that there is an optimum radius of the cation for which diffusivity is maximum.

\section{Methods}

\subsection{Interionic potential}

We have employed the modified Parinello-RahmanVashistha (PRV) inter-ionic pair potential of Shimojo et al. ${ }^{1}$ This potential function consists of four terms i.e., a soft core repulsive term, a Coulomb term, a charge-dipole term and a van der Waal's term.

$$
V_{i j}=\frac{H_{i j}}{r^{n_{i j}}}+\frac{Z_{i} Z_{j} e^{2}}{r}-\frac{1}{2}\left(P_{i j}\right) \frac{e^{2}}{r^{4}}-\frac{W_{i j}}{r^{6}},
$$

where

$$
\begin{gathered}
H_{i j}=A_{i j}\left(\sigma_{i}+\sigma_{j}\right)^{n_{i j}}, \\
P_{i j}=\alpha_{i} Z_{j}^{2}+\alpha_{j} Z_{i}^{2} .
\end{gathered}
$$

Here the polarizability of the cation is assumed to be zero. This is because the difference in sizes of cation
Table 1. Interionic potential parameters employed in the present study for AgI system. All the parameters are given in units of $14.39 \mathrm{eV}$ and $\AA$, and have been taken from Shimojo and Kobayashi. ${ }^{1}$

\begin{tabular}{lccc}
\hline & $\mathrm{Ag}^{+}-\mathrm{Ag}^{+}$ & $\mathrm{Ag}^{+}-\mathrm{I}^{-}$ & $\mathrm{I}^{-}-\mathrm{I}^{-}$ \\
\hline$n_{i j}$ & 11 & 9 & 7 \\
$H_{i j}$ & 0.011282 & 90.971 & 370.06 \\
$P_{i j}$ & 0.0 & 1.0353 & 2.0706 \\
$W_{i j}$ & 0.0 & 0.0 & 5.8659 \\
\hline
\end{tabular}

and anion is large and therefore the contribution due to the cation is insignificant compared to the anion. The first term gives the repulsive contribution and the last term the attractive dispersion contribution. The second term accounts for the Coulombic interaction and the third term is the polarizability term. Here $i, j$ denote type of ions (cation/anion), $\mathrm{A}_{i j}$ is the repulsive strength, $\sigma_{i}, \sigma_{j}$ are ionic radii, $\alpha_{i}, \alpha_{j}$ are electronic polarizabilities and $\mathrm{Z}_{i}, \mathrm{Z}_{j}$ are the fractional charges on the ions. Electronic polarizability of $\mathrm{Ag}^{+}$is assumed to be zero by virtue of its smaller size. $n_{i j}$ is the degree of hardness of the repulsive interaction between the ions $i$ and $j .{ }^{20}$ The parameters for the above interionic potential are given in table 1

In the present study, we carried out MD simulations for different cation sizes by varying $\sigma_{i}$ for the cation $\mathrm{Ag}^{+}$while keeping all other parameters unchanged. Ewald summation was carried out to calculate the Coulomb term in the pair potential. A cut off distance of $15 \AA$ has been employed.

\subsection{Simulation details}

Simulations were carried out on a system consisting 864 ions $\left(432 \mathrm{Ag}^{+}\right.$and $432 \mathrm{I}^{-}$) in a cubic box of length $31.1784 \AA$ corresponding to a density of $5.55 \mathrm{gm} / \mathrm{cc}$. Our starting configuration is b.c.c. lattice in which iodine ions were placed on a b.c.c. lattice and $\mathrm{Ag}^{+}$ions were placed in the tetrahedral voids. The radius of the cation $\left[\left(\sigma_{i}\right.\right.$ in equation (2)) $]$ has been varied between 0.33 and $0.68 \AA$ while the anion radius $\left(\sigma_{j}=\sigma_{I^{-}}\right)$has been taken as $2.2 \AA$. This corresponds to cation to anion radius ratio of $0.15-0.309$. We note that this is smaller than the limiting octahedral void radius ratio of 0.414 but encompasses the tetrahedral void radius ratio of 0.225 . 0.155 is the minimum radius ratio for the formation of the stable ionic mixture. ${ }^{27}$ The system was equilibrated at $1000 \mathrm{~K}$ in the microcanonical ensemble for $1 \mathrm{~ns}$ followed by a production run of $2 \mathrm{~ns}$. During the latter period, we stored positions, velocities and forces of all the ions for further analysis at every $25 \mathrm{fs}$. A time 
step of 2 fs ensured us good conservation in the total energy $\left(1\right.$ in $\left.10^{5}\right)$. We carried out simulations with the DLPOLY simulation package. ${ }^{28}$

\section{Results and discussions}

\subsection{Diffusivity as a function of ionic radii}

In figure 1 , the $\mathrm{Ag}^{+}-\mathrm{Ag}^{+}, \mathrm{Ag}^{+}-\mathrm{I}^{-}$and $\mathrm{I}^{-}-\mathrm{I}^{-}$radial distribution functions(rdf) along with their coordination numbers are shown. Figure $1 \mathrm{a}$, where the $\mathrm{Ag}^{+}-\mathrm{Ag}^{+}$ radial distribution functions for different cation sizes are shown, suggest that the $\mathrm{Ag}^{+}$ions are disordered in position. The rdf appears similar to that for a fluidlike phase. The position of the first peak varies between $4.1 \AA$ and $4.3 \AA$ for different cation sizes and the coordination number varies between 4 and 13 . There are small differences in the height of the first peak depending on the cation radii. For the cation radii of $0.53 \AA$, the $g(r)$ is 1.357 at the position of the maximum in the first peak which occurs at $4.325 \AA$. For $0.33 \AA$ cation radii, the $\mathrm{g}(\mathrm{r})$ is somewhat higher at 1.369 at $r=4.125 \AA$. $5.5 \AA$ is where the minimum is seen for $0.33 \AA$ cation which shifts to $6.05 \AA$ for $0.53 \AA$. The decrease in the $g(r)$ value for $0.53 \AA$ shows that the $\mathrm{Ag}^{+}-\mathrm{Ag}^{+}$correlation actually decreases for $0.53 \AA$ as compared to $0.33 \AA$. For the $\mathrm{Ag}^{+}-\mathrm{I}^{-}$rdf, the position of the first maximum is near $2.25 \AA$ for $0.33 \AA$ cation radius and the $g(r)$ value is 4.58 . For $0.53 \AA$ cation, the first maximum is near $2.51 \AA$ with a value of $g(r)$ is 3.75 . These changes suggest that the correlation between $\mathrm{Ag}^{+}$and $\mathrm{I}^{-}$actually decreases on going from $0.33 \AA$ to $0.53 \AA$. The minimum between the first two shells occurs at $3.53 \AA$ and the value of rdf is 0.51 for $0.33 \AA$ as compared to $3.87 \AA$ and a value of rdf of 0.58 for $0.53 \AA$. Thus, for $0.53 \AA$, the rdf at the maximum of the first peak is lower than for $0.33 \AA$ and the minimum has a value that is higher than the $0.33 \AA$. As a result, the rdf for the $0.53 \AA$ is more fluid-like showing weaker oscillations. In addition to these changes, we notice that the position of the first peak maximum shifts to larger values of $r$ as the cation radius increases. For $\mathrm{I}^{-}-\mathrm{I}^{-}$rdf, trends are similar to what has been seen for $\mathrm{Ag}^{+}-\mathrm{Ag}^{+}$rdf where the first peak maximum is lower for $0.53 \AA$ as compared to $0.33 \AA$ but at the same time the value of rdf at the minimum between the first and second peak is not higher for the $0.53 \AA$ radius cation. Thus these changes are somewhat ambiguous and do not indicate clearly that the $0.53 \AA$ cation is indeed more fluid-like. But the rdf between $\mathrm{Ag}^{+}$and $\mathrm{I}^{-}$clearly suggests that the $0.53 \AA$ rdf is more fluid-like. Later we shall see that the $0.53 \AA$ cations are associated with higher diffusivity.
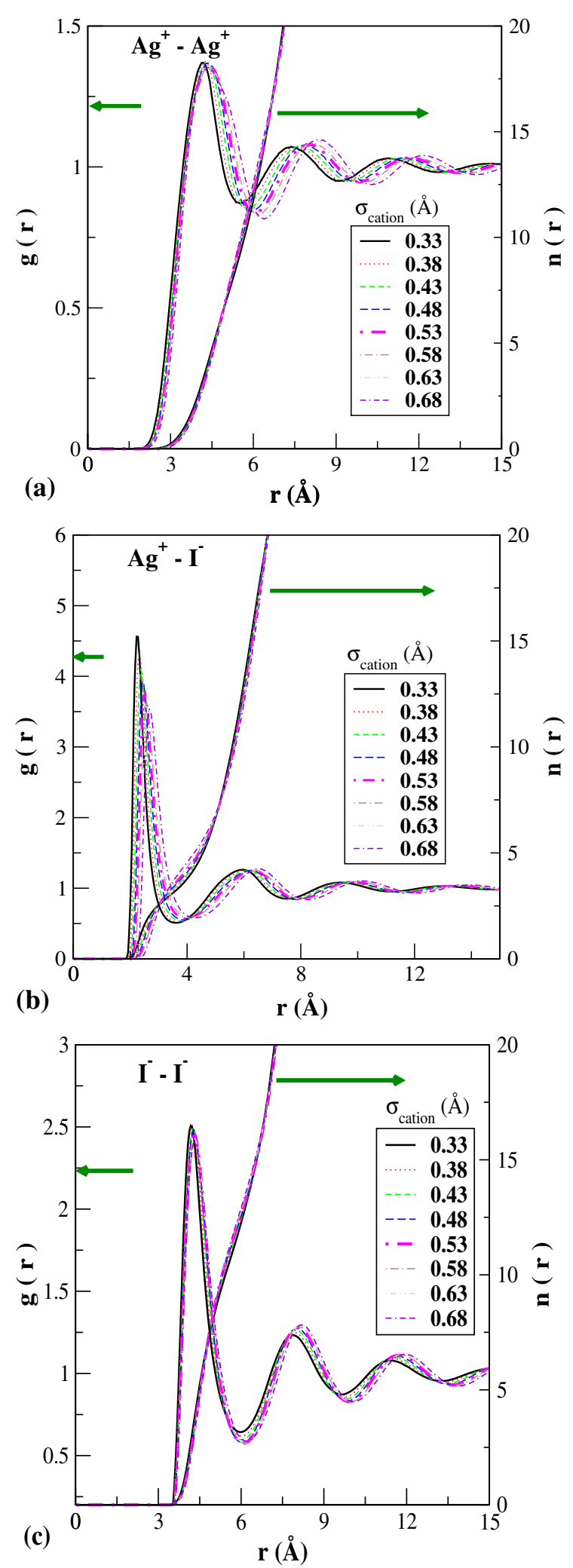

Figure 1. Radial distribution functions (rdfs) between (a) $\mathrm{Ag}^{+}-\mathrm{Ag}^{+}$, (b) $\mathrm{Ag}^{+}-\mathrm{I}^{-}$and (c) $\mathrm{I}^{-}-\mathrm{I}^{-}$in molten $\mathrm{AgI}$ at $1000 \mathrm{~K}$. The corresponding coordination numbers are shown on the scale on the right side of each plot. The curves corresponding to $0.33 \AA$ and $0.53 \AA$ are drawn with thick lines. 


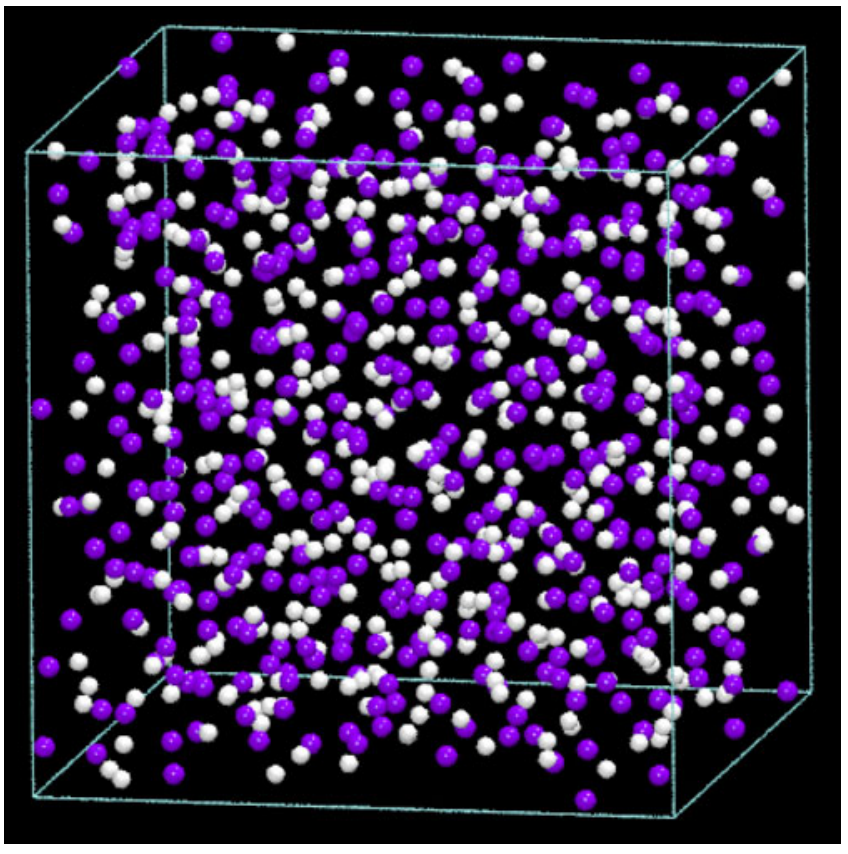

Figure 2. Snapshot of molten $\mathrm{AgI}$ at $1000 \mathrm{~K}\left(\mathrm{Ag}^{+}\right.$: white spheres, $\mathrm{I}^{-}$: violet or grey (in black and white) spheres.

In figure 2, we provide a snapshot of the molten $\mathrm{AgI}$ at $1000 \mathrm{~K}$. It is clear from the figure that $\mathrm{Ag}^{+}$and $\mathrm{I}^{-}$ are disordered and distributed uniformly within the unit cell. There is no clustering of these ions. The anionic sub-lattice has indeed melted and the $\mathrm{I}^{-}$ions are not located at b.c.c lattice positions is also evident from the figure.

Mean square displacement (MSD) of cations of different sizes are shown in figure $3 \mathrm{a}$. On increase in the cationic radii from $0.33 \AA$ onwards the slope of MSD increases. The maximum slope is seen for cation of size $0.53 \AA$. Further increase in cationic radii leads to a decrease in the slope of the MSD. Figure $3 b$ shows a plot of diffusivity as a function of cation radii. The maximum in diffusivity is seen for cation of $0.53 \AA$ radius. For sizes beyond $0.53 \AA$, there is a rapid decrease in diffusivity. The values of diffusivities are of the order of $10^{-8} \mathrm{~m}^{2} / \mathrm{s}$. These are rather high and clearly correspond to superionic regime. The error bars in the calculation of diffusivity is about $2 \%$. Thus, the changes in $\mathrm{D}$ with cation radius is beyond the error bar. These results are in excellent agreement with the trends seen by Flygare and Huggins. ${ }^{18}$ They studied $\alpha$-phase of AgI where $\mathrm{I}^{-}$forms a b.c.c sub lattice. They further varied cation radii and found that cation radii of $0.83 \AA$ has maximum diffusivity. They carried out a minimum energy path analysis as a function of cationic radii. They further reported a number of other quantities of interest, such as activation energy. The activation energy was found to be a minimum for the cation of radius $0.83 \AA$. We have also computed diffusivity of $\mathrm{I}^{-}$ion for different cationic radii. The diffusivities of $\mathrm{I}^{-}$ion corresponding to different cation radii are $0.337(0.33 \AA), 0.3145(0.38 \AA)$, $0.2909(0.43 \AA), \quad 0.2651(0.48 \AA), \quad 0.2739(0.53 \AA)$, $0.264(0.58 \AA), 0.2319(0.63 \AA), 0.23(0.68 \AA)$. It is seen that there is a near-continuous decrease in diffusivity of $\mathrm{I}^{-}$ion with increase in cationic size. This is shown in figure 4. A slight increase is seen at cation radius of $0.53 \AA$ probably because of the strong influence of the cation motion on the anion and association between the
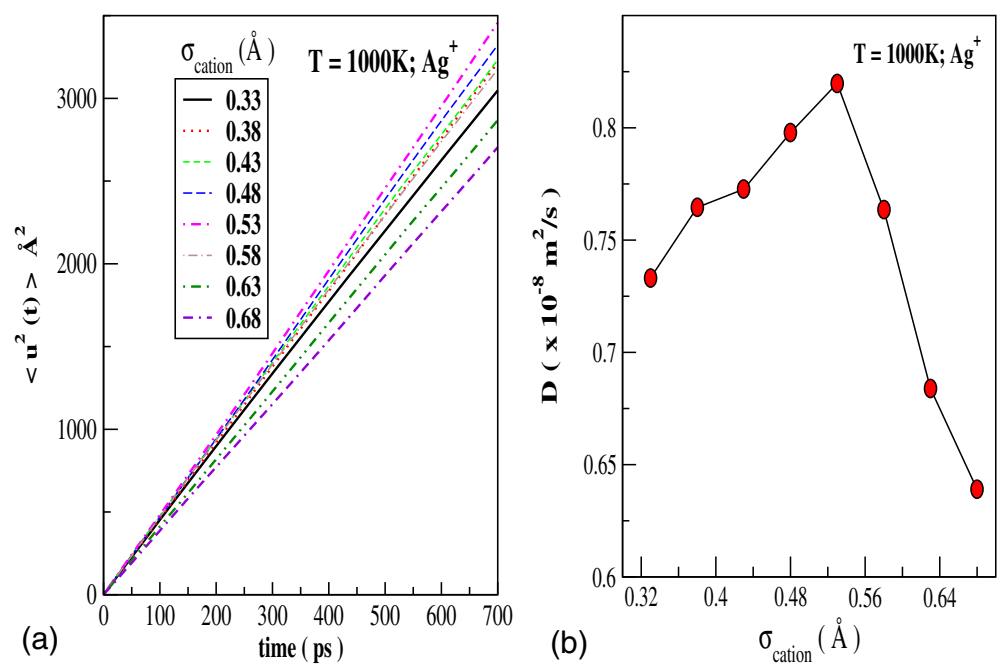

Figure 3. (a) Mean square displacement of cation for different sizes (curves corresponding to $0.33 \AA$ and $0.53 \AA$ are drawn with thick lines). (b) Self-diffusivity of cation as a function of its radius (diffusivity shows a maximum at $0.53 \AA$ ). 
cation and the anion. However, there is no significant maximum seen for the anion like the one seen for the cation.

Earlier studies from this laboratory suggest that irrespective of the condensed matter phase studied, there exists a diffusivity maximum as a function of radius or diameter of the diffusant. Studies in porous solids such as zeolites, dense liquids, dense close packed solids, dense amorphous solids as well as ions in polar solvents all exhibit a similar diffusivity maximum. ${ }^{24}$ The effect of this can be seen in changes in entropy as a function of diffusant radius as suggested originally by Kemball. ${ }^{29}$ Derouane and co-workers later showed that these changes arise from the mutual cancellation of forces exerted on the diffusant by the medium to which it is confined. ${ }^{30-32}$ Here we see a similar maximum in molten iodide. Origin of this maximum in molten iodide also arises from mutual cancellation of forces leading to levitation of particles and is therefore known as 'Levitation Effect(LE)'. We now report other properties in order to understand the nature of the diffusivity maximum.

The ionic conductivity of the molten salt can be obtained from the current-current correlation function:

$$
\begin{aligned}
J(t) . J(0) \\
=\left\langle\left(\sum_{i} v_{i+}(t)-\sum_{j} v_{j-}(t)\right)\right. \\
\left.\cdot\left(\sum_{i} v_{i+}(0)-\sum_{j} v_{j-}(0)\right)\right\rangle .
\end{aligned}
$$

The current-current correlation function is a collective quantity which is the sum of self and crosscorrelation terms. ${ }^{33-35}$ Due to the poor statistics arising from a single value obtained for the whole system, it is difficult to obtain it accurately. It also depends on the reference system and use of barycentric reference system avoids long time fluctuations in the correlation function. ${ }^{36}$ The conductivity is given by

$$
\sigma=\frac{e^{2}}{3 V k_{B} T} \int_{0}^{\infty} J(t) . J(0) d t
$$

where $V$ is the volume of the simulation cell, $k_{B}$ is the Boltzmann constant, $T$ is the temperature and $e$ is the magnitude of the charge on the electron. The selfdiffusivity and the electrical conductivity are related through the relation

$$
\sigma=\frac{N e^{2}}{2 V k_{B} T}\left(D_{+}+D_{-}\right)(1-\Delta),
$$

where $N$ is the total number of ions, $D_{+}$is the diffusivity of the cations and $D_{-}$is the diffusivity of the anions. $\Delta$ is usually a positive constant.

In figure 5, the velocity auto correlation function(VACF) for the cations of size 0.33 and $0.53 \AA$ are shown. Cation of radius $0.33 \AA$ exhibits a more intense back scattering as compared to the cation of size $0.53 \AA$. This is surprising since $0.53 \AA$ cation is larger and should have encountered greater number of collisions. The weaker back scattering of $0.53 \AA$ arises from the weaker undulations in the energy of the trajectory associated with this cation. Indeed Flygare and Huggins ${ }^{18}$ found lower undulations in the loci of minimum energy path of cation associated with maximum diffusivity. In their case they studied the superionic phase where the maximum in diffusivity is found for $0.83 \AA$. In figure 5 , we also show the power spectrum obtained from the Fourier transform of the velocity autocorrelation functions. Such lower undulations found in the
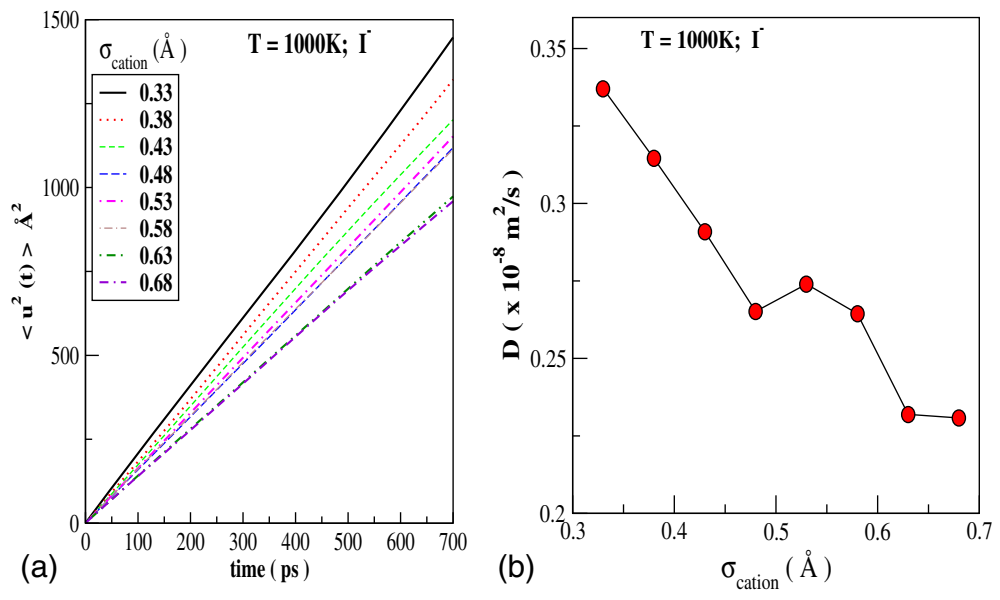

Figure 4. (a) Mean square displacement of anion for different sizes of cation. (b) Self-diffusivity of anion as a function of the radius of the cation is shown. 

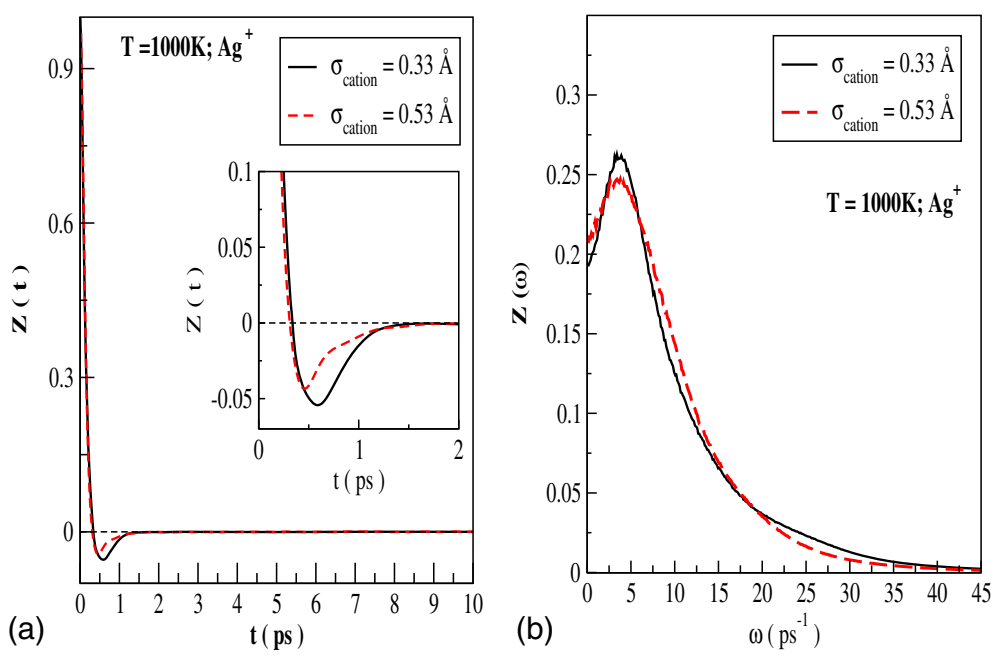

Figure 5. (a) Velocity autocorrelation function (VACF) for cation radii $0.33 \AA$ and $0.53 \AA$. Larger back scattering is seen for $0.33 \AA$ (see inset). (b) Power spectrum for cation radii $0.33 \AA$ and $0.53 \AA$ (higher value at zero frequency seen for $0.53 \AA$ as compared to $0.33 \AA$ is evident and is consistent with the diffusivity values shown in plot of diffusivity against ionic radii (see figure $3 \mathrm{~b}$ ))

potential energy profile as it traverses through the ionic melt should translate to lower activation energy as suggested by Truhlar. ${ }^{37}$ In order to check if this is indeed the case we have computed the activation energies by carrying out simulations at four different temperatures. The nominal (actual) temperatures for $0.53 \AA$ guest is are $900 \mathrm{~K}(903.7 \mathrm{~K}), 950 \mathrm{~K}(961.1 \mathrm{~K}), 1000 \mathrm{~K}(1009.6 \mathrm{~K})$, $1050 \mathrm{~K}(1054.8 \mathrm{~K})$ while for $0.33 \AA$ the corresponding nominal temperatures are 895.7, 940.0, 985.5 and $1039.3 \mathrm{~K}$. The diffusivities of cation of size $0.33 \AA(0.53 \AA)$ at these temperatures are $0.617(0.677)$, $0.679(0.734), 0.731(0.821), 0.817(0.876) \mathrm{m}^{2} / \mathrm{s}$, respectively. Figure $5 \mathrm{~b}$ shows the Fourier transform of the velocity autocorrelation function. The value at zero frequency is higher for $\sigma_{\text {cation }}=0.53 \AA$ which is consistent with the diffusivity values obtained from the MSD. Arrhenius plots for these two cations are shown in figure 6. The activation energy for $0.33 \AA(0.53 \AA)$ is $16.2(14.0) \mathrm{kJ} / \mathrm{mol}$. This clearly demonstrates that the higher diffusivity is associated with lower activation energy and vice versa.

Figure 7 a shows the logarithm of the self part of intermediate scattering function $\left(\mathrm{F}_{s}(\mathrm{k}, \mathrm{t})\right)$ for somewhat small wave number, $\mathrm{k}=0.2 \AA^{-1}$. This wave number corresponds to a length of the order of simulation cell length, $\approx 31 \AA$. The curves are shown for cations of sizes $0.33 \AA$ and $0.53 \AA$. It is clear from the figure that $\ln \left(\mathrm{F}_{s}(\mathrm{k}, \mathrm{t})\right)$ for both these cations have similar slopes up to $50 \mathrm{ps}$. Beyond 50 ps, the slope decreases for $0.33 \AA$ suggesting that this cation is associated with at least two different relaxation processes. In contrast, the $0.53 \AA$ ion exhibits a single slope through out the first $250 \mathrm{ps}$. This is consistent with our earlier findings ${ }^{24}$ where a single exponential decay was seen for the diffusant at diffusivity maximum. We have also calculated relaxation times $(\tau)$ associated with these processes from the slope of the curve. The two $\tau$ values for the cation of

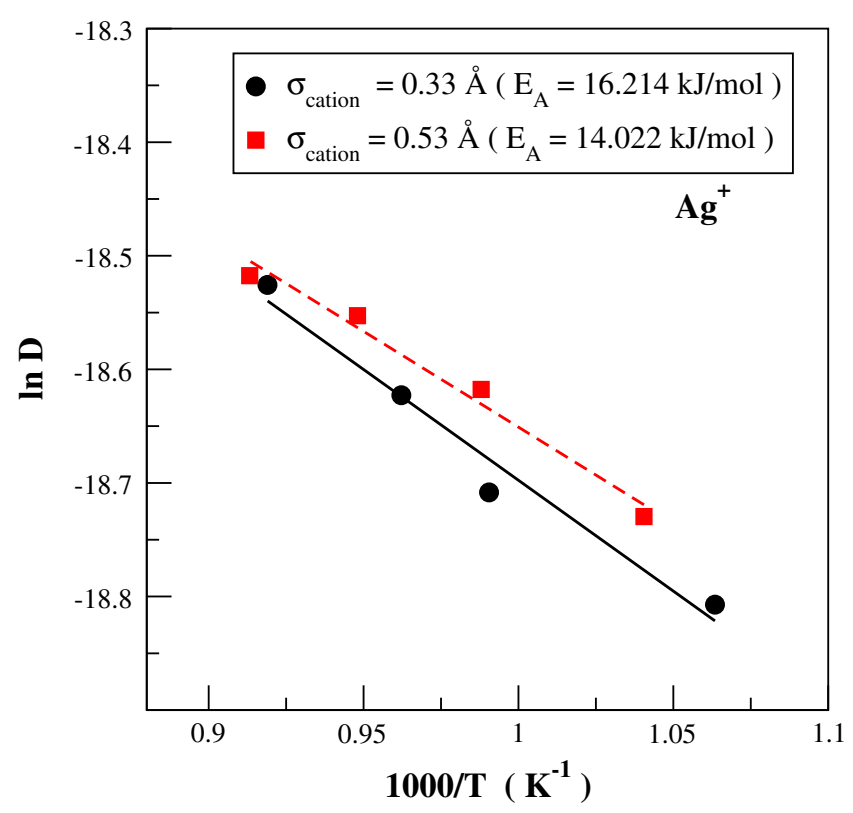

Figure 6. Arrhenius plot of self-diffusivity for the cation is shown for two cation sizes for molten AgI. The lower activation energy of the larger cation of $0.53 \AA$ suggests that the anomalous dependence of diffusivity on cation radius arises from mutual cancellation of forces. 

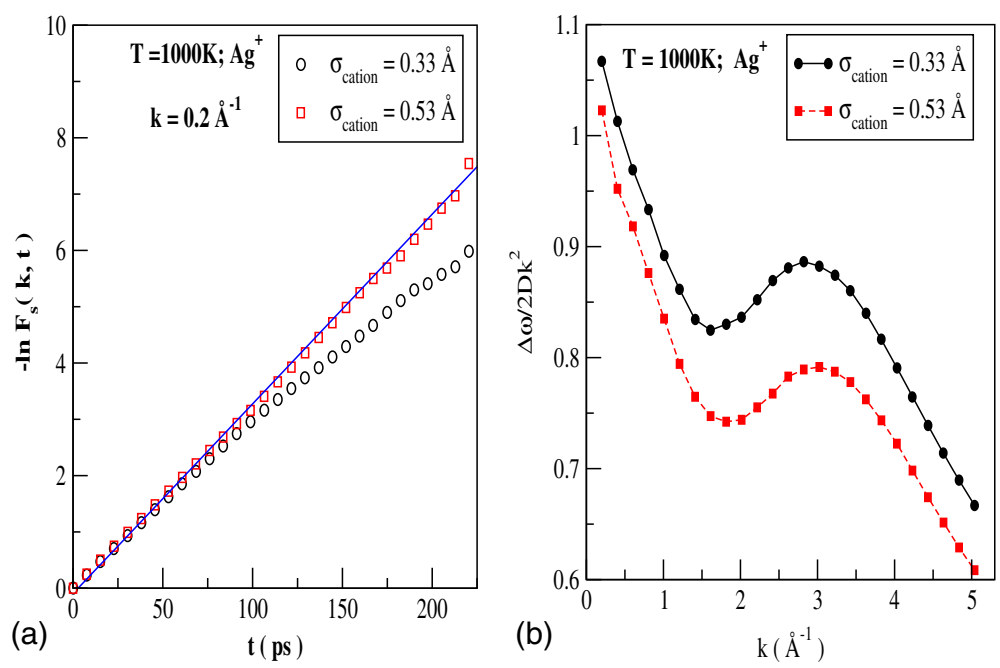

Figure 7. (a) Logarithm of intermediate scattering function $\mathrm{F}_{s}(\mathrm{k}, \mathrm{t})$ for $k=0.2 \AA^{-1}$. Note that a change of slope occurs for $0.33 \AA$ cation after 50 ps. A straight line for the $0.53 \AA$ cation between $1-250$ ps shows that the slope does not change for this cation. (b) Wave number dependence of full width at half maximum or FWHM of dynamic structure factor, $\mathrm{S}_{s}(\mathrm{k}, \omega)$ obtained by Fourier transformation of $\mathrm{F}_{s}(\mathrm{k}, \mathrm{t}) .0 .53 \AA$ cation shows weaker undulations suggesting that this cation is associated with lower energy barrier during diffusion which is consistent with the lower activation energy, see previously.

radii $0.33 \AA$ are 30.98 ps and 56.00 ps whereas for the cation of size $0.53 \AA \tau$ is 30.90 ps. Kawakita et al. ${ }^{38}$ report quasi-elastic neutron scattering measurements on molten cuprous iodide. They report the dynamic structure factor at $650^{\circ} \mathrm{C}$. Further, they obtain the intermediate scattering function by Fourier transformation of the dynamic structure factor. They concluded that the relaxation time of the density-density correlation reveals that molten $\mathrm{CuI}$ exhibits extremely slow dynamics with the characteristic relaxation time of 1.606 ps at $0.86 \AA^{-1}$, which is much longer than the self-diffusion of $\mathrm{Cu}$ ions.

We have also calculated the self part of the dynamic structure factor $\left(\mathrm{S}_{s}(\mathrm{k}, \omega)\right)$ by taking the Fourier transform of the intermediate scattering function $\left(\mathrm{F}_{s}(\mathrm{k}, \mathrm{t})\right)$. In figure $7 \mathrm{~b}$, we show the full width at half maximum (FWHM) of $S_{s}(\mathrm{k}, \omega)$ as a function of wave number. The full width at half maximum (FWHM, $\Delta \omega$ ) of dynamic structure factor is related to translational motion of the ion. The value of this quantity at the hydrodynamic limit is $2 \mathrm{Dk}^{2}$, where $k$ is the wave vector. To see the dynamic behaviour of the ion at different length scales, we have plotted the ratio between $\Delta \omega$ and its value at hydrodynamic limit as a function of wave number(k) (see figure 7b). From the figure, we can see that there is a minimum followed by a maximum. In the present case both $0.33 \AA$ and $0.53 \AA$ ions are showing a minimum followed by a maximum. These undulations in the curve are actually density correlations in the medium which are associated with higher energetic barriers for translational motion of the diffusant through it. More intense undulations will be associated with large energetic barrier. It is evident from the figure that the undulations are more for $0.33 \AA$ cation as compared to $0.53 \AA$ cation which means that $0.33 \AA$ cation encounters an energetic barrier that is larger than that of $0.53 \AA$ which is also clear from the values of activation energies. Also to be noted is that the curve for $0.53 \AA$ decays faster than for $0.33 \AA$ cation which is consistent with the higher self-diffusivity of $0.53 \AA$ as compared to $0.33 \AA$.

\section{Conclusions}

Our molecular dynamics results on molten AgI show an anamolous increase in diffusivity with an increase in cationic radii. The diffusivity maximum occurs at $0.53 \AA$ cation radius. This behaviour has similarities with levitation effect(LE) which has been seen in many condensed matter phases. The cation size at the diffusivity maximum has lower activation energy as compared to the smaller cation sizes. This is in accordance with the predictions of levitation effect. Also this is in agreement with the findings of Flygare and Huggins. ${ }^{18}$ 


\section{References}

1. Shimojo F and Kobayashi M J 1991 Phys. Soc. Jpn. 60 3725

2. Chandra S 1981 Superionic solids principles and application (Amsterdam: North-Holland)

3. GoodEnough J B 1984 Proc. R. Soc. Lond. A 393215

4. Tallon J L 1988 Phys. Rev. B 389069

5. Wood B C and Marzari N 2006 Phys. Rev. Lett 97 166401

6. Hull S 2004 Rep. Prog. Phys. 671223

7. Bitrin V and Trulls J 2007 J. Chem. Phys. 126021105

8. Ivanov-Shitz A K 2007 Crystallogr. Reports 52302

9. Nakamura K, Ihata K, Yokoyama Y, Nomura K and Kobayashi M 2001 Ionics 7178

10. Hull S, Berastegui P and Grippa A 2005 J. Phys. Cond. Matt. 171067

11. Hull S, Keen D A, Madden P A and Wilson M 2007 J. Phys. Cond. Matt. 191

12. Alcaraz O and Trullas J 2001 J. Chem. Phys. 1157071

13. Yokoyama Y and Kobayashi M 2003 Solid State Ionics 15979

14. Ivanov-Schitz A K, Mazniker B and Povolotskaya E 2003 Solid State Ionics 15963

15. Vashishta P, Ebbsjo I, Dejus R and Skold K 1985 J. Phys. C: Solid State Phys. 18291

16. Rino J P, Yvone M M, Hornos C, Giomal A, Antonio C, Ebbsjo I, Kalia R K and Vashishta P 1988 J. Chem. Phys. 897542

17. Kumar P P and Yashonath S 2006 J.Chem. Sci. 118135

18. Flygare W H and Huggins R 1973 J. Phys. Chem. Solids 341199

19. Parinello M and Rahman A 1980 Phys. Rev. Lett. 45 1196
20. Parinello M, Rahman A and Vashishta P 1983 Phys. Rev. Lett. 501073

21. Vashishta P and Rahman A 1978 Phys. Rev. Lett. 40 1340

22. Kumar P P and Yashonath S 2002 J. Am. Chem. Soc. 124 3828

23. Ghorai P K and Yashonath S 2005 J. Phys. Chem. 109 3979

24. Yashonath S and Ghorai P K 2008 J. Phys. Chem. B 112 665

25. Roselieb K and Jambon A 2002 Geochim. Coschim. Acta 66109

26. Huang S H, Yoshida F, You J, Jiang G and Xu K 1999 J. Phys. Cond. Matt. 115429

27. Pauling L 1929 J. Am. Chem. Soc. 511010

28. Forester T R and Smith W 1985 The DL-POLY-2.0 Reference Manual, version 2.0 (CCLRC, Daresbury Laboratory: Warrington, U.K.)

29. Kemball C 1950 Adv. Catal. 2233

30. Derouane E G 1987 Chem. Phys. Lett. 142200

31. Derouane E G, Andre J M and Lucas A 1988 J. Catal. 11058

32. Derycke I, Vigneron J, Lambin P, Lucas A and Derouane E G 1991 J. Chem. Phys. 944620

33. Hansen J and McDonald I 1975 Phys. Rev. A 112111

34. Agarwal M, Ganguly A, Chakravarty C 2009 J. Phys. Chem. B 11315284

35. Agarwal M and Chakravarty C 2009 Phys. Rev. E 79 030202(R)

36. Kamala C, Ayappa K and Yashonath S 2002 Phys. Rev. E 65061202

37. Truhlar D G 1978 J. Chem. Edu. 55309

38. Kawakita Y, Tahara S, Fujii H, Kohara S and Takeda S 2007 J. Phys.: Cond. Matt. 19335201 